\title{
surfinpy: A Surface Phase Diagram Generator
}

\author{
Adam R. Symington ${ }^{1}$, Joshua Tse ${ }^{2}$, Marco Molinari ${ }^{2}$, Arnaud \\ Marmier $^{3}$, and Stephen C. Parker ${ }^{1}$
}

1 Department of Chemistry, University of Bath 2 Department of Chemistry, University of Huddersfield 3 FET - Engineering, Design and Mathematics, University of the West of England

DOI: $10.21105 /$ joss. 01210

\section{Software}

- Review 七

- Repository ¿

- Archive [

Submitted: 25 January 2019

Published: 20 February 2019

\section{License}

Authors of papers retain copyright and release the work under a Creative Commons Attribution 4.0 International License (CC-BY).

\section{Summary}

A surface phase diagram is a graphical representation of the different physical states of a surface under different conditions. The surface represents the first point of contact between the material and the environment. Thus, understanding the state of surface is crucial for a wide range of problems in materials science concerning the relationship between the state of the surface and the surrounding environmental condtions. Examples include particle morphologies in solid state batteries (Canepa et al., 2018); determining the concentration of adsorbed water at a surface depending on synthesis conditions (Molinari, Parker, Sayle, \& Islam, 2012) (Tegner, Molinari, Kerridge, Parker, \& Kaltsoyannis, 2017); catalytic reactions (Reuter \& Scheffler, 2003); or determing the effect of dopants and impurities on the surface stability.

Computational modelling can be used to generate surface phase diagrams from energy minimisation data. One common research question is how water adsorption affects the surface and material properties. The conventional starting point is to perform a series of energy minimisation calculations with varying concentrations of water on several different slabs. From the energies, the surface free energy of each calculation (phase) as a function of temperature and pressure can be calculated using a well-established approach (Molinari et al., 2012). Once the free energy is known under different constants, the phase which is most stable at a specific temperature and pressure, and thus a phase diagram, can be generated.

A further degree of complexity can be introduced by considering surface defects, e.g., vacancies or interstitials, or other adsorbants, e.g., carbon dioxide. Using surface defects as an example, it is important to consider the relationship between the defective surface, the stoichiometric surface and the adsorbing water molecules. A surface phase diagram can be constructed as a function of the chemcial potential of the adsorbing species (water) and the surface defect (e.g., oxygen, if oxygen vacancies are being considered). This is done using the method of Marmier \& Parker(2004).

\section{surfinpy}

surfinpy is a Python module for generating surface phase diagrams from DFT data. It contains two core modules for generating surface phase diagrams using both the methods employed in Molinari et al. (2012) and Marmier \& Parker (2004). These allow fast generation of temperature vs. pressure phase diagrams and phase diagrams as a function of chemcial potential of species A and B. The plotting classes take the outputs of the calculation modules and generate phase diagrams using matplotlib. surfinpy is aimed 
(a)

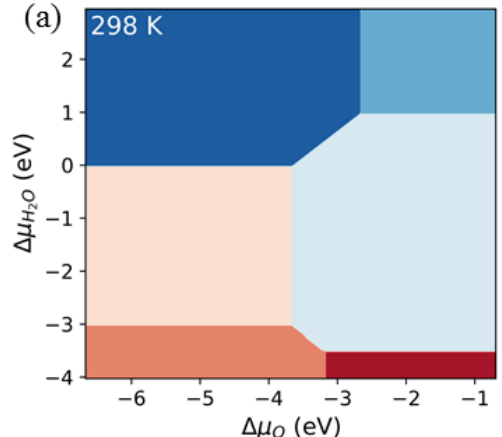

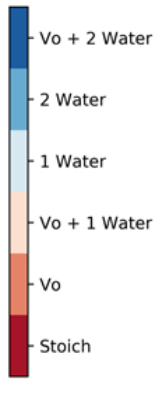

Figure 1: An example phase diagram as a function of chemical potential (a), and as a function of temperature and pressure (b).

towards theoretical solid state physicist who have a basic familiarity with Python. The repository contains examples of the core functionality as well as tutorials, implemented in Jupyter notebooks to explain the full theory. Furthermore, a detailed description of theory is also available within the documentation.

\section{Acknowledgements}

ARS would like to thank Andrew R. McCluskey for his guidance through this project. This package was written during a PhD funded by AWE and EPSRC (EP/R010366/1). The input data for the development and testing of this project was generated using ARCHER UK National Supercomputing Service (http://www.archer.ac.uk) via our membership of the UK's HEC Ma-terials Chemistry Consortium funded by EPSRC (EP/L000202).

\section{References}

Canepa, P., Dawson, J. A., Sai Gautam, G., Statham, J. M., Parker, S. C., \& Islam, M. S. (2018). Particle morphology and lithium segregation to surfaces of the li7la3zr2o12 solid electrolyte. Chemistry of Materials, 30(9), 3019-3027. doi:10.1021/acs.chemmater. 8 b00649

Marmier, A., \& Parker, S. C. (2004). Ab initio morphology and surface thermodynamics of $\alpha-\mathrm{Al}_{2} \mathrm{O}_{3}$. Phys. Rev. B, 69(11), 115409. doi:10.1103/PhysRevB.69.115409

Molinari, M., Parker, S. C., Sayle, D. C., \& Islam, M. S. (2012). Water adsorption and its effect on the stability of low index stoichiometric and reduced surfaces of ceria. The Journal of Physical Chemistry C, 116(12), 7073-7082. doi:10.1021/jp300576b

Reuter, K., \& Scheffler, M. (2003). First-principles atomistic thermodynamics for oxidation catalysis: Surface phase diagrams and catalytically interesting regions. Phys. Rev. Lett., 90(4), 046103. doi:10.1103/PhysRevLett.90.046103

Tegner, B. E., Molinari, M., Kerridge, A., Parker, S. C., \& Kaltsoyannis, N. (2017). Water adsorption on ano2 111, 110, and 100 surfaces $(\mathrm{an}=\mathrm{u}$ and $\mathrm{pu})$ : A density functional theory + u study. The Journal of Physical Chemistry C, 121(3), 1675-1682. doi:10.1021/ acs.jpcc.6b10986 